\title{
The Clinical Relevance of Cerebral Microbleeds
}

Review Article

Karadi ZN, Csecsei P, Lovig C, Lovadi EI, Szapary L*

Department of Neurology, University of Pecs Clinical Centre, Hungary.

Abstract

Despite their very recent discovery and only couple decade-long existence, cerebral microbleeds are progressively investigated, trending radiological concepts in today's scientific era. In this paper, we are going to provide a short review on the most important details of cerebral microbleeds, with special focus on their clinical relevance and particular emphasis on their practical aspects with stroke prevention and therapy.

Keywords: Cerebral Microbleed; Magnetic Resonance Imaging; Stroke; Cognitive Impairment; Small Vessel Disease.

\section{Introduction}

In the last couple decades, the rapid evolution of imaging methods shed light on previously unknown entities. These findings confirmed discoveries from as early as the nineteenth century, when forms of small vessel disease were first documented.

Today, these entities have gained an important role in daily clinical practice, due to the fact that not solely the immensely developed technology can depict them, therefore clearing the way for expansive research. There has been numerous investigations for the better understanding of the radiological and histopathological concepts of cerebral microbleeds; but recently, most studies have been focusing on their clinical relevance such as cognitive impairment and a possible role as an imaging marker for brain hemorrhage.

\section{Cerebral Microbleeds Definition and Imaging}

Cerebral microbleeds are radiologically defined lesions visible on magnetic resonance imaging (MRI). They were first documented simultaneously by Chan, Greenberg and Offenbacher in 1996 [1]. The detailed pathology behind the lesions were described in 1999 by Fazekas as cerebral amyloid deposition and lipohyalinosis in cases of intracerebral hemorrhage [2], however the very same year Tanaka published another explanation pointing out the role of damaged arteriosclerotic microvessels [3]. Most studies agree that microbleeds indicate sites of accumulated hemosiderincontaining macrophages $[2,3]$ that arise owing to the submerged transport system from the iron-rich breakdown of minute hemorrhages from the damaged network of vessels [4]. Once they become visible, they scarcely disappear: the low rate of export of hemosiderin suggests a small number of true resorption and the dominance of possible artifacts [5]. Other suggestions of their origin include a hypothesis that they are results of parallel pro-inflammatory pathways activated by vasculopathy [6]; and a preposition presenting that age-related disorders of ferritin transport and storage might take notable part in microbleed pathomechanism [7].

Cerebral microbleeds are small, round, homogenous, hypointense lesions depicted by gradient recall echo (GRE) or susceptibility weighted (SWI) imaging (Figure 1) [8]. Both methods provide increased contrast between the paramagnetic material and brain tissue [9], however SWI is proved to be 50-70 percent more efficient than the GRE sequence, and currently the most sensitive in detecting microbleeds. Combining high-pass filtered phase information with images of multiplied magnitude $[8,10$, 11]; contributes to augmented contrast and increased blooming effect $[8,10]$. The appearance of cerebral microbleeds is highly dependent on imaging parameters including the sequence itself, the spatial resolution, magnetic field strength and the post-processing [8]. Studies have reported a 30 percent rise in identification of microbleeds with 3.0T field strength compared to $1.5 \mathrm{~T}$ [12],

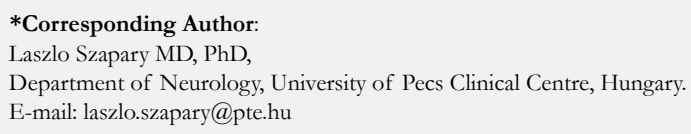

Copyright: Szapary $\mathbf{L}^{\mathcal{O}}$ 2016. This is an open-access article distributed under the terms of the Creative Commons Attribution License, which permits unrestricted use, distribution and reproduction in any medium, provided the original author and source are credited. 
Figure 1. Imaging of Cerebral Microbleeds with Susceptibility Weighted Imaging.

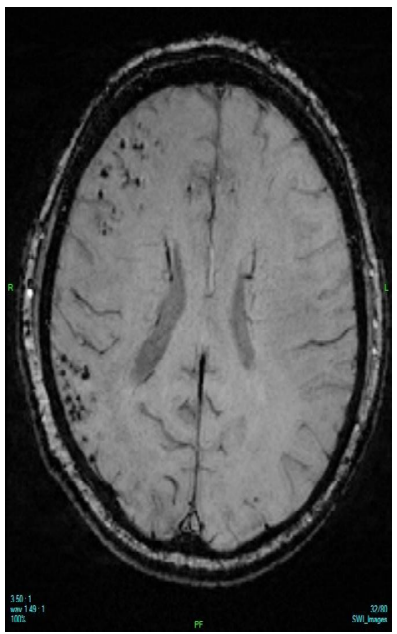

The small, homogenous, hypointense, round lesions are located in the lobar area in our patient from the Stroke Care Unit.

moreover, the ultra-high resolution 7.0T field strength MRI seems to overcome these results [10]. Not solely the microbleed number but size is also contingent on these imaging parameters, and due to the blooming effect, MRI results are unable to measure the exact size of the lesions. Cerebral microbleeds are often bimodal in their size distribution, ranging from $2 \mathrm{mms}$ to $5-10 \mathrm{mms}$ with a cut-off at $5.7 \mathrm{mms}[1,8,13]$.

The neuroimaging concensus criteria for detecting cerebral microbleeds involve the following key points: (1) homogenous foci on GRE or SWI, (2) round or ovoid in shape, (3) small in size - mainly 2-5 to $10 \mathrm{mms}$, (4) they present with blooming effect, (5) they devoid signal hyperintensities, (6) surrounded by brain parenchyma, (7) diffuse axonal injury and secondary causes are excluded and (8) microbleed mimics are ruled out $[1,8]$.

These microbleed mimics refer to various sites of calcification or iron deposition (mainly choroid plexus, basal ganglia and the pineal gland, cross-sectional vessels in the sulci, deoxyhemoglobin containing structures, air in the sinuses and bone artifacts along with secondary causes, particularly vascular malformations, metastatic tumors and diffuse axonal injury [8]. Differential diagnosis requires other imaging sequences to rule out the non-hemorrhagic lesions, artifacts and secondary phenomena that appear identically to microbleeds. In order to minimize misdiagnosis due to coexisting mimics, the standard imaging for cerebral microbleeds should include SWI or GRE, T1, T2, FLAIR and DWI sequences along with conventional head CT $[1,8]$.

Currently, manual rating scales, including Brain Observer MicroBleed Scale (BOMBS) and Microbleed Anatomical Rating Scale (MARS) are proved to be useful in clinical settings. BOMBS categorizes microbleeds by their size, side and location (deep, lobar, posterior) [14]. The MARS scale provides a slightly more sophisticated approach primarily classifying microbleeds as 'definite' or 'possible', then registering the side and the location similarly to the BOMBS method except for the size, which appears to be unnecessary in the scaling system (Figure 2). These alterations offer a good to very good inter- and intrarater variablilty of the microbleed assessment [15]. Automated detection methods are also available, however despite their very high sensitivity, they are overly deficient in specifity; making manual evaluation the gold standard [16]. However semi-automated methods requiring visual censor for false positives would reshape current time-consuming procedures $[16,17]$.

\section{Cerebral Microbleeds in the General Population}

The Rotterdam Study has been a pioneer in the research of cerebral microbleeds. Over twelve thousand brain scans have been processed since the beginning of the study in 1995 and almost 4000 scans were evaluated for cerebral microbleeds [18]. $15.3 \%$ of the study population had microbleeds, including $5.4 \%$ with multiple microhemorrhages. Age seems to play significant role in the appearance of microbleeds: they were detected in $6.5 \%$ of the patients between the age of 45 and 50, which increased to $35.7 \%$ among individuals over 80 years of age $[18,19]$.

This research group also suggested that microbleeds could demonstrate the worsening of the underlying brain lesions, therefore a longitudinal study including 831 patients was additionally performed. The mean interval between examinations was 3.4 years, whilst $10.2 \%$ of the patients developed new microbleeds, increasing the overall prevalence from $24.4 \%$ to $28 \%$. If baseline microhemorrhages were present, new microbleeds appeared with the odds ratio of 5.36; moreover, this number escalated to 7.15 if multiple microhemorrhages were present. This study found that besides their number, microbleeds at baseline also predicted location; two thirds of the newly evolved microbleeds were strictly lobar $[5,18]$.

The preferential distribution of cerebral microbleeds reflect the primary pathology. Microhemorrhages are generally classified topographically as lobar, deep and infratentorial (Figure 3) [20, 21]. Lobar microbleeds are associated with cerebral amyloid angiopathy, $\beta$-amyloid deposition and certain APOE genotypes $[2,21]$. Lesions in the deep or infratentorial regions are preferably relate to vascular risk factors, mainly hypertension [19, 21]. Areas above the corpus callosum are most presumably due to traumatic injuries, while lesions in the thalamus and the basal ganglia imply to non-traumatic sources [22].

The tremendous number of patients involved in the population- 
Figure 2. The Microbleed Anatomical Rating Scale (MARS).

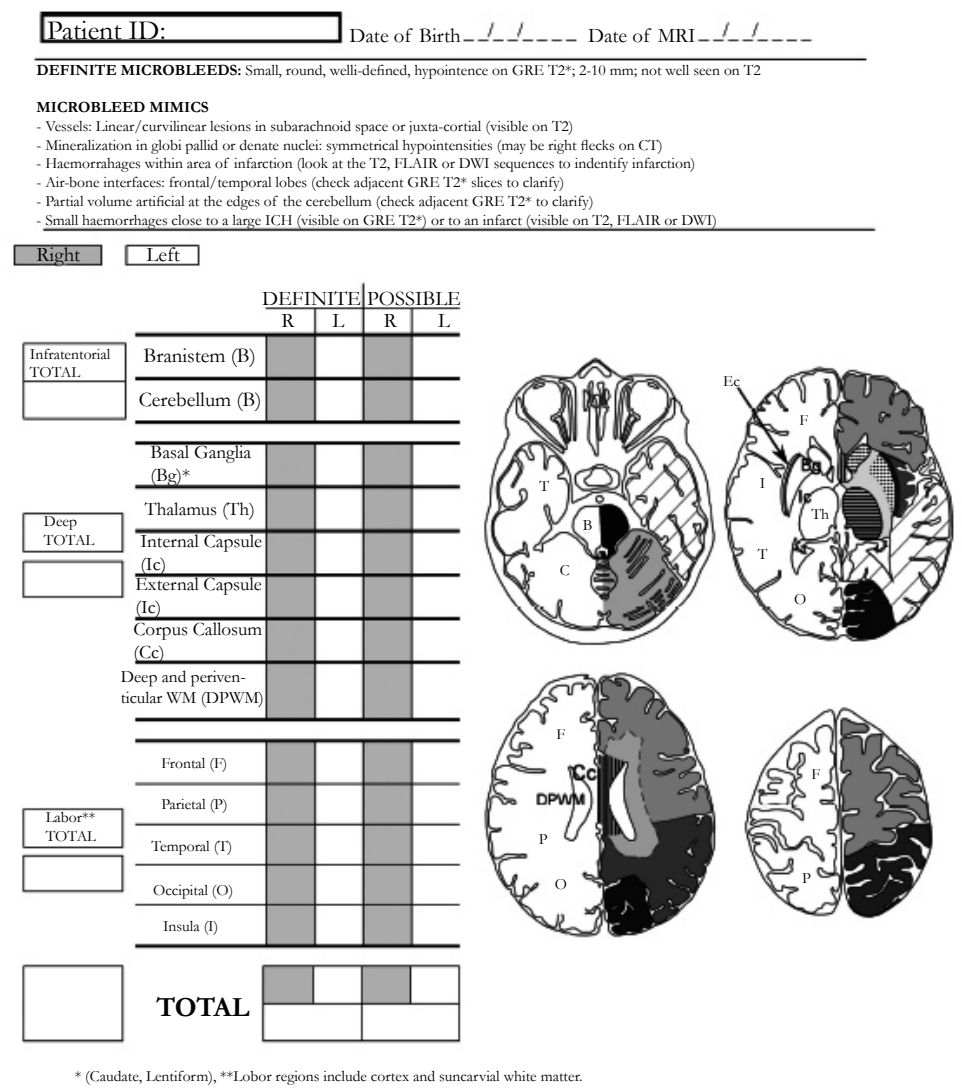

Gregoire et al., suggested a new, reliable concept by the subdivision of the microbleeds to probable and definite lesions as the main concept of the scale. These microhemorrhages are then classified as deep, infratentorial or lobar on each side [15].

Figure 3. Topography of the Cerebral Microbleeds.

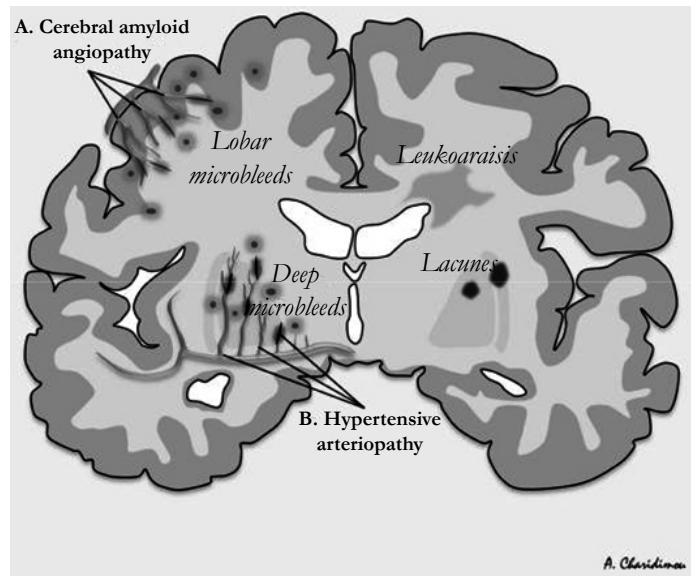

Location of the lesions indicate the underlying pathology, as deep microbleeds are results of hypertension or chronic vascular risk factors and deep microhemorrhages are caused by cerebral amyloid angiopathy [95].

based studies produced information on the risk factors of cerebral microbleeds: age, gender, hypertension, low cholesterol levels, statin therapy, retinal microvascular lesions, left ventricular hypertrophy and APOE 4 genotype, the latter showing strong correlation to strictly lobar microhemorrhages [5, 19, 23, 24]. The role of the other alleles on APOE gene remain controversial as APOE 2 allele appears to cause fibrinoid necrosis, which contributes to the formation of microbleeds, however statistics do not verify this hypothesis $[19,25,26]$. Microbleeds are also commonly associated with genetic conditions including cerebral autosomal dominant arteriopathy with subcortical infarcts and leukoencephalopathy (CADASIL) and Notch-3 protein mutation $[27,28]$; polymorphysms of neprilysin $[9,29]$ and the complement receptor-1 gene [30]; mutations of collagen 4 1-subunit [31], APP and presenilin [32]; Fabry's disease [33], familial multiple cavernoma syndrome and Tangier's disease [34]. Cerebral microhemorrhages my appear in cases of fat [35] and septic embolism [36], infective endocarditis [37], cerebral vasculitis [38], moyamoya disease [39] and they are also closely related to other subtypes of small vessel disease $[40,41]$. 


\section{Small Vessel Disease}

Microbleeds, as the hemorrhagic margin of a spectrum disease, are often found together with other pathologies, such as subcortical microinfarcts, lacunes, white matter hyperintensities and focal brain atrophy $[41,42]$. They are all key features of small vessel disease, one of the most common pathologies in the brain, a heterogenous systemic defect that affects various organs, commonly the small vessels in the brain [41]. Similarly to microbleeds, other parenchymal lesions are detectable by highresolution MRI methods, however small vessel disease itself cannot be visualized in vivo, allowing imaging results to function as radiological markers [42].

A consensus for classification named STandards for ReportIng Vascular changes on neuroimaging (STRIVE) was created to structure a clear system of neuroimaging protocols and consistent terminology. The new, standardized classification and reporting method relies on individual and clinical judgement besides strict imaging criteria, providing a useful guideline to both research and routine clinical practice [41].

Not only the appearance but the origin of these lesions is also heterogenous: main etiological categories include arteriolosclerosis (mainly associated with age, hypertension, diabetes mellitus), cerebral amyloid angiopathy, genetic factors (CADASIL, and other inherited diseases), inflammatory or immunological disorders, venous collagenosis and further secondary causes [42].

Major factors in the pathomechanism are vessel wall damage, change of cerebral blood flow and therefore chronic ischemia, disturbance of the blood-brain barrier, inflammation and apoptosis of oligodendrocytes [42]. Newer studies show importance of capillary disfunction as a shared marker of small vessel disease with special focus on pericytes, as key elements [43].

Cerebral amyloid angiopathy (CAA) is a condition of amyloidosis or amyloid deposition of the cerebrovascular system [44, 45]. Although several types of accumulating proteins have been described, amyloid $\beta$-protein is by far the most common type with major clinical relevance. CAA is associated with aging - appearing in half of the elderly population, it also plays an important role in Alzheimer's disease where amyloid $\beta$-protein depostition is found in more than $80 \%$ of the patients [46]. Its effect on intracerebral hemorrhage is still the focus of intensive research. CAA-associated cerebral vasculopathies contribute to microbleeds and intracranial hemorrhage in lobar, especially occipital location; indicating that microhemorrhages preferably occur where $\beta$-amyloid deposition is utmost [48]. In a study, cerebral microhemorrhages were present in almost half of the cases of confirmed CAA [49]. The inclusion of microbleeds into the Boston criteria [50], the validated clinical diagnostic approach of CAA in intracerebal hemorrhage appear to increase sensitivity of detecting CAA [8, 34, 40]. Nevertheless, recent data, based on 113 cases, has queried if this finding reflects the background of all microbleeds and presented results that show no significant correlation in distribution and the affected sites between CAA and lobar microbleeds, implying that no direct neuropathological association is present between the two entities [51].

\section{Cerebral Microbleeds and Clinical Relevance}

Although cerebral microbleeds have been described as asymptomatic lesions, emerging data points out that these lesions are able to generate clinical syndromes if appearing rapidly or manifesting at important sites [52]. Apart from their presence in healthy individuals $[5,19,23]$, cerebral microbleeds are also related to ischemic and hemorrhagic strokes [2, 53], cognitive impairment and Alzheimer's disease [54] along with diffuse axonal injury associated with traumatic brain injury [55].

\section{Cerebral Microbleeds and Stroke}

Microhemorrhages reflect a bleeding prone state, therefore it is not unforeseen that they are found in $19-83 \%$ of cases of intracerebral hemorrhages [9]. Also, the number and location of the lesions are proportional to the risk and volume of hemorrhages $[8,56]$. While a single microbleed merely means $14 \%$ risk of developing further symptomatic hemorrhages in three years, more than six microlesions increase the likelihood to $50 \%$, serving as an indicator for the risk of recurrence $[40,57]$ However, these facts do not suggest that micro - and macrohemorrhages are related; on the contrary, despite sharing features to some extent, they are entirely different entities [13].

Charidimou reported increased risk of intracerebral hemorrhage but not ischemic events when microbleeds were present [58], although several studies proved that they are not solely associated with conditions susceptible to bleeding, and they are also present in $15-35 \%$ of the ischemic strokes [9]. Findings remain controversial as a European investigation discovered no increased risk of hemorrhage risk but probability of future ischemic lesions, while a Japanese study stated strong relation to intracerebral bleedings rather than ischemic events [58]. Incident microbleeds point to the progression of ischemic vascular lesions, particularly if multiple lesions are present [59]. Moreover, they appear even in patients with transient ischemic attack (TIA) where they tend to emerge less [53], but they are linked to a higher risk of progression into stroke [60]. There is also a four-fold increased likelihood of detecting microhemorrhages in cases of recurrent stroke events. [9] Lim et al., reported that in TIA, recurrent strokes occured in only $4.2 \%$ in patients without microhemorrhages, and they were considerably associated with strictly lobar and mixed microbleeds, at rates $14.3 \%$ and $38.5 \%$, respectively [61]. It has also been revealed that the lesions at mixed locations relate to increased risk of developing stroke after TIA in a manner independent of the number of the lesions [61].

The progression of the underlying pathologies are undeniably reflected by the number of microbleeds: $13 \%$ increase was recorded in the first four days and $23 \%$ in the next five years after a stroke event [62]. Another study measured the rate of the formation of new microbleeds in the three following years after TIA or ischemic stroke and found an extra 0.8 microlesions per year on average. Furthermore, the number of microbleeds were increased by 5.4 lesions per year in patients with more than five microbleeds at baseline [63].

The coexistence of ischaemic and hemorrhagic events in the presence of microbleeds implies that these microlesions function as markers of both first ever intracerebral hemorrhages and 
ischemic strokes in healthy individuals [63, 64], as well as suggesting increased risk of recurrent hemorrhage after intracerebral hemorrhage [57] and among survivors of ischemic stroke [65]. Cerebral microbleeds are associated with higher risk to developing a future episode of stroke in general, representing both ischemic and hemorrhagic consequences; with a dose-dependent manner based on the number of microhemorrhagical lesions [57, 59, 66]. Cerebral microbleeds at regions generally affected by CAA imply an increased risk of intracerebral hemorrhage, while sites without CAA may reflect both ischemic and hemorrhagical alterations as the underlying stroke pathology [64].

Based on several previously mentioned studies, it has become undoubtful that presence and even number of microbleeds at baseline, along with radiological signs of previous cerebrovascular events mark a notable relation to the shaping of new microbleeeds that contribute to recurrent strokes [67]. Therefore, cerebral microbleeds emerge as potential biomarkers for the increased risk of any types of cerebrovascular events [66], preparing the way for a possibly revolutionary method of diagnostics in clinical practice.

\section{Cerebral Microbleeds and Thrombolysis}

Thrombolytic therapy is proved to improve the three-month functional outcome if administered in a short time-window in acute stroke [68]. Despite its undeniable merit, secondary post-ischemic symptomatic intracranial bleeding generated dilemma in decisionmaking in the acute phase of ischemic stroke [69-72], leading to very strict inclusion criteria and a list of contraindications; even so these guidelines only contain standard radiological

information without microbleeds [73]. Thrombolysis-associated hemorrhagic transformation was found in a one-week time period in $6.8 \%$ of patients receiving intravenous recombinant tissue plasminogen activator (rTPA) [72, 73].

Despite numerous attempts, there are no valid predictive scores able to filter patients more likely to develop secondary hemorrhagic transformation [72]. The risk of secondary hemorrhage might be related to age, blood pressure, early changes in diffusion imaging and ischemic signs on CT and severity of stroke [74], however cerebral microbleeds might serve as more reliable markers for risk associated with thrombolysis in stroke patients [69, 72] since their number increases rapidly after administration of rTPA [75]. However, it still remains unknown if microhemorrhages contribute to secondary bleedings after intravenous thrombolysis [76].

Numerous studies showed no significant relation even between multiple microbleeds and intravenous thrombolysis-related complications [69, 71, 76-79]. Until further data is available on their relation, the presence of cerebral microbleeds are not contraindication for the use of rTPA, therefore they are not included in thrombolysis guidelines.[71-72]

\section{Cerebral Microbleeds and Antithrombotic Therapy}

Analogously to the previously discussed rTPA treatment, bleeding, especially intracranial hemorrhage is a rare, but the most feared complication of antithrombotic therapy [19, 80, 81]. These drugs have become the center of attention in acute clinical settings, since decision-making is extremely burdensome when costbenefit balance is taken into consideration; emerging the need for risk stratification for potentially life-threatening intracerebral hemorrhages [82]. Besides pointing to the development of hemorrhagic strokes, cerebral microbleeds are also markers of antiplatelet and anticoagulant-related intracerebral bleedings [19, $42,81,83-85]$.

Antiplatelets are increasingly used medications in prevention and treatment of cardiovascular, cardiometabolic and cerebrovascular diseases. Presence of baseline microhemorrhages had a significant correlation with symptomatic brain hemorrhages in antiplatelet users, the relation was even more conspicuous when multiple lesions were present $[81,84,86,87]$. New formation of cerebral microhemorrhages was indicated by baseline number of lesions [5], classic vascular risk factors including age, blood pressure, gender, diabetes mellitus and lipid status $[21,88]$ and microangiopathy $[5,88]$. Lovelock et al reported relation between microbleeds and antiplatelet therapy in patients with intracerebral bleeding but not with TIA or ischemic stroke [83], however other studies revealed the same correlation regarding ischemic cerebrovascular lesions $[58,82]$. The Rotterdam Study revealed that aspirin might be only associated with strictly lobar lesions, emphasizing the importance of the underlying small vessel disease [81]. Other studies investigating effects of clopidogrel demostrated that clopidogrel use was associated with presence and even higher number of microbleeds in a stroke-free population [89], however, only a nonsignificant correlation was found in patients with strokev [90]. Moreover, in contrast to aspirin, clopidogrel was more related to deep and infratentorial lesions than lobar ones [90, 91].

Some investigations suggested that the increased risk of intracerebral hemorrhage might outweigh the benefit of antiplatelets as methods of secondary prevention [85, 88]. Nevertheless, the lack of significant association between microbleeds and antiplatelets in stroke-free population [82] questions the need for overriding current guidelines.

Vernooij et al. found in the Rotterdam study that microbleeds were only related to antiplatelet drugs but not to anticoagulants [81], that would be explained by a hypothesis suggesting that platelet aggregation is a more important factor in microbleed evolution than clot formation [81]. Other studies seems to be in contrast to this findings as they reported higher percent of patients developing new microbleeds when baseline lesions were present than in microbleed-negative cases [92]. Microhemorrhages have also been proved to increase in stroke patients with prior anticoagulant therapy [93]. Akoudad et al., located most of the microbleeds in the deep and infratentorial area in stroke-free patients on coumarin treatment [94], while other studies reported association with lobar lesions [95]. It has been verified that presence of microhemorrhages and prothrombin time, particularly high variability in international normalized ratio (INR) values are independent contributors to intracerebral hemorrhage in patients treated with warfarin $[94,96]$. An impaired hemostatic system with inability to remain self-limited therefore causing microbleeds to expand, could be a general explanation for higher prevalence of both microbleeds and intracerebral hemorrhages [95, 97].

Novel oral anticoagulants (NOAC) have proved to lower risk of intracerebral bleeding by almost 50\% [98-101]. Saito et al., 
found no new microbleeds in the NOAC group in contrast with the warfarin group, where the conventional anticoagulation was independently associated with the appearance of new lesions [102]. These results might be attributable to a more stable hemostatic state with NOAC, since studies focusing on conventional anticoagulation showed that warfarin treatment with well-balanced and optimal INR range have similar risk of hemorrhage to NOAC therapy [95].

Similarly to questions regarding antiplatelet-safety, the costs versus the benefits of anticoagulants are even more intensely argued, since they are key elements for treatment of cardiovascular diseases, especially for the primary and secondary stroke prevention in non-valvular atrial fibrillation, as they manage to lower risk by almost $70 \%$ [103]. Several study results raise awareness to the use of anticoagulants in patients with microbleeds, and therefore researchers argue whether oral anticoagulants still have the net benefit when the the risk of potentially disabling hemorrhage is so high [57] that statistically, prevention of a small number of cardioembolic strokes costs even higher number of hemorrhages in patients treated with warfarin [104]. Despite these findings, Haley et al., presented that based on a decision model, only as high as a 13-fold increased risk of intracerebral hemorrhage would balance benefits of conventional anticoagulant use [105]. Several suggestions have been published for a potential screening algorithm for high-risk patients (Figure 4), however in the absence of randomized trials, these proposals remained in the background. $[95,106]$.

\section{Cerebral Microbleeds and Cognitive Impairment}

The relation between microbleeds and cognitive performance have been intensively researched [42]. Despite the fact that these lesions were believed to be silent, clinical and populationbased studies have reported symptomatic manifestations of neurovascular damage $[42,107]$. Small vessel disease is one the

Figure 4. Decision Algorithm for the Anticoagulation of High-Risk Patients.

MRI Screening for Anticoagulation
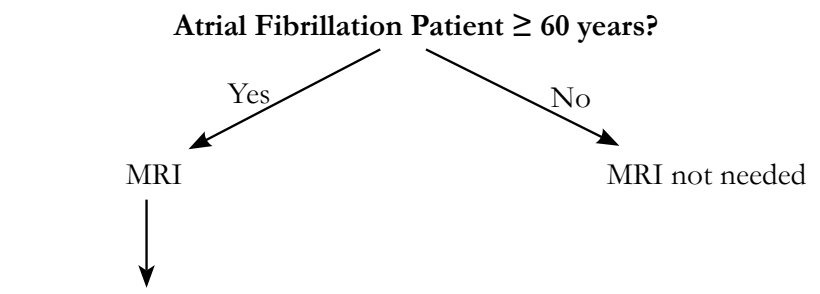

Microbleeds Present ?

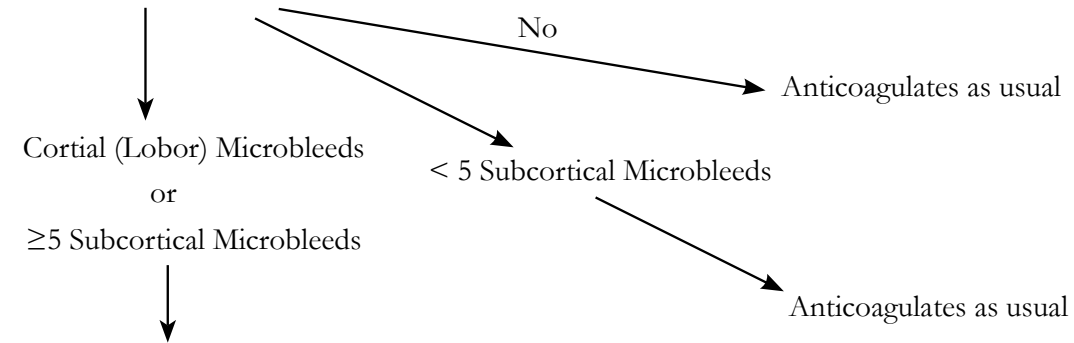

1. Neurology Consultation

2. For anticoagulation, avoid warfarin in favor of newer anticoagulants
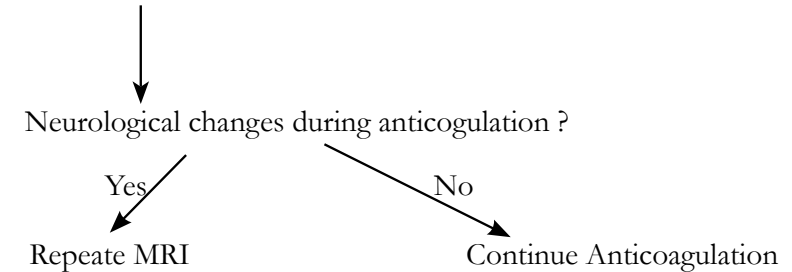

Continue Anticoagulation

High morbidity and mortality of the potential hemorrhagic complication of the antithrombotic drugs emerge the need for careful clinical decision-making. Flow charts and risk assessments before treatment might offer a safer but not profitable alternative. [Fisher M (2013) MRI screening for chronic anticoagulation in atrial fibrillation. Front. Neurol. 4:137. doi: 10.3389/ fneur.2013.00137.] 
most common source and also the marker of progression of vascular dementias, since underlying pathomechanisms might include CAA or hypertensive arteriopathy, especially that it has also been suggested that these lesions may be able to limit cerebral plasticity $[42,108]$.

Cerebral microbleeds are found in up to $19 \%$ in the cogntitively unaffected population, however they are detected in higher percents in mild cognitive impairment, Alzheimer's disease and vascular dementia $(20-43 \%, 18-32 \%$ and $65-85 \%$, respectively) [9]. The presence of microhemorrhages was associated with lower scores on the Mini Mental State Examination (MMSE) or other neuropsychological testing methods $[109,110]$. The RUNDMC study however, revealed that microbleeds were linked to worse cognitive function without change in MMSE scores [111].

It was revealed in the Rotterdam Study that deep microbleeds are not significantly related to cognitive outcome, however they affect global cognition, psychomotor speed, gait and attention. Lobal lesions are associated with memory and executive functions besides global cognition [9]. The baseline number of microhemorrhages were proportional to risk of cognitive impairment: a single microbleed marked a $16 \%$ risk, while more than six lesions reflected a notable increase to $52 \%$ risk for cognitive dysfunction [57]. Presence of cerebral microbleeds reflected an overall twofold risk for dementia [112]. Results from other studies do not support the relation between microhemorrhages and cognitive impairment [113].

\section{Cerebral Microbleeds and Prognosis}

Kim et al. investigated morbidity and prognosis among stroke patients with cerebral microhemorrhages. They documented a strong relation between poor functional outcome and microbleeds, especially the ones located in the infratentorial region [114]. They suggest that microbleeds and white matter hyperintensities indicate sites of chronic dysfunction in perfusion and reactivity, contributing to more critical damage in the penumbra area, leading to limited recovery $[114,115]$. Prognosis is also influenced by the location affected by the lesions, since focal damages in important sites might worsen functional outcome [114].

Not only the location but the number of microhemorrhages are also related to prognosis, especially in bleeding-prone conditions: it was found in patients after thrombolysis that pre-existing microbleeds were associated with disease outcome [116], furthermore microbleed number was independently linked to increased score on the modified Rankin scale [117]. Besides direct effects of these microlesions on cognition and neurologic dysfunction, effects of secondary hemorrhages after thrombolysis or the administration of antithrombotic drugs worsen functional outcome in stroke patients [117].

The PROSPER study focuses on the connection between microhemorrhages and mortality in patients with cerebrovascular disease, antithrombotic therapy or cardio- and cerebrovascular risk factors. It is demonstrated that microbleeds are the most linked to overall mortality among imaging markers. They have found that overall mortality is higher in the group with more than one microbleed compared to the group without microbleeds present [118], analogously to a memory clinic study where the same result was found in patients with more than three microbleeds [119]. Multiple microbleeds also beared a six-fold increase in stroke-related mortality, and when investigating strictly lobar, CAA-related lesions, a 7.2-fold risk for stroke-associated deaths was revealed without any connection to cardiovascular mortality. Similarly to microbleed-related morbidity, their affect on mortality is also contingent of the number of cerebral microbleeds [118].

\section{Conclusion}

Along with the technical development of imaging methods, cerebral microbleeds are getting increasing attention both at scientific research and clinical practice. They have become particularly important for their increasing burden on health. Despite the intensive investigation, results remain highly controversial, thus relevant information for the treatment and clinical guidelines is missing. Our paper reveals highly inconsistent findings among recent studies of cerebral microbleeds. However, it has been proposed that these differences might no longer exist if all imaging and patient selection methods could be standardized. Methodological consistency is even more important considering that microbleeds are primarily radiological concepts, where slight differences in depicting could result in extreme divergence of results.

Emerging data on the relation between cerebral microbleeds and cognitive impairment is already in the spotlight as these lesions were previously believed to be related to no symptomatic complications. Ongoing trials, namely, the CROMIS-2 [120] or the CMB-NOW [121] might provide so far lacking data that expectantly contribute to further understanding of cerebral microbleed pathology, pathomechanism and clinical impications.

\section{References}

[1]. Cordonnier C, Al-Shahi Salman R, Wardlaw J (2007) Spontaneous brain microbleeds: systematic review, subgroup analyses and standards for study design and reporting. Brain. 130(8): 1988-2003.

[2]. Fazekas F, Kleinert R, Roob G, Kleinert G, Kapeller P, et al., (1999) Histopathologic analysis of foci of signal loss on gradient-echo T2*-weighted MR images in patients with spontaneous intracerebral hemorrhage: evidence of microangiopathy-related microbleeds. AJNR Am J Neuroradiol. 20(4): 637-642.

[3]. Tanaka A, Ueno Y, Nakayama Y, Takano K, Takebayashi S (1999) Small chronic hemorrhages and ischemic lesions in association with spontaneous intracerebral hematomas. Stroke. 30(8): 1637-142.

[4]. Kleinig TJ (2013) Associations and implications of cerebral microbleeds. J Clin Neurosci. 20(7): 919-927.

[5]. Poels MM, Ikram MA, van der Lugt A, Hofman A, Krestin GP, et al., (2011) Incidence of cerebral microbleeds in the general population: the Rotterdam Scan Study. Stroke. 42(3): 656-661.

[6]. Menon RS, Burgess RE, Wing JJ, Gibbons MC, Shara NM, et al., (2012) Predictors of highly prevalent brain ischemia in intracerebral hemorrhage. Ann Neurol. 71(2): 199-205.

[7]. Janaway BM, Simpson JE, Hoggard N, Highley JR, Forster G, et al., (2014) Brain haemosiderin in older people: pathological evidence for an ischaemic origin of magnetic resonance imaging (MRI) microbleeds. Neuropathol Appl Neurobiol. 40(3): 258-269.

[8]. Greenberg SM, Vernooij MW, Cordonnier C, Viswanathan A, Al-Shahi Salman R, et al., (2009) Cerebral microbleeds: a guide to detection and interpretation. Lancet Neurol. 8(2): 165-174.

[9]. Yates PA, Villemagne VL, Ellis KA, Desmond PM, Masters CL, et al., (2013) Cerebral microbleeds: a review of clinical, genetic, and neuroimaging associations. Front Neurol. 4: 205.

[10]. Nandigam RN, Viswanathan A, Delgado P, Skehan ME, Smith EE, et al., (2009) MR imaging detection of cerebral microbleeds: effect of susceptibility-weighted imaging, section thickness, and field strength. AJNR Am J 
Neuroradiol. 30(2): 338-343

[11]. Goos JD, van der Flier WM, Knol DL, Pouwels PJ, Scheltens P, et al., (2011) Clinical relevance of improved microbleed detection by susceptibility-weighted magnetic resonance imaging. Stroke. 42(7): 1894-900.

[12]. Tatsumi S, Ayaki T, Shinohara M, Yamamoto T (2008) Type of gradient recalled-echo sequence results in size and number change of cerebral microbleeds. AJNR Am J Neuroradiol. 29(4): e13.

[13]. Greenberg SM, Nandigam RN, Delgado P, Betensky RA, Rosand J, et al., (2009) Microbleeds versus macrobleeds: evidence for distinct entities. Stroke. 40(7): 2382-2386.

[14]. Cordonnier C1, Potter GM, Jackson CA, Doubal F, Keir S, et al., (2009) Improving interrater agreement about brain microbleeds: development of the Brain Observer MicroBleed Scale (BOMBS). Stroke. 40(1): 94-99.

[15]. Gregoire SM, Chaudhary UJ, Brown MM, Yousry TA, Kallis C, et al., (2009) The Microbleed Anatomical Rating Scale (MARS): reliability of a tool to map brain microbleeds. Neurology. 73(21):1759-66.

[16]. Fazlollahi A, Meriaudeau F, Giancardo L, Villemagne VL, Rowe CC, et al., (2015) Computer-aided detection of cerebral microbleeds in susceptibilityweighted imaging. Comput Med Imaging Graph. 46 (3): 269-76.

[17]. Kuijf HJ, Brundel M, de Bresser J, van Veluw SJ, Heringa SM, et al., (2013) Semi-Automated Detection of Cerebral Microbleeds on 3.0 TMR Images. PLoS One. 8(6): e66610.

[18]. Ikram MA, van der Lugt A, Niessen WJ, Koudstaal PJ, Krestin GP, et al., (2015) The Rotterdam Scan Study: design update 2016 and main findings. Eur J Epidemiol. 30(12): 1299-315.

[19]. Poels MM, Vernooij MW, Ikram MA, Hofman A, Krestin GP, et al., (2010) Prevalence and risk factors of cerebral microbleeds: an update of the Rotterdam scan study. Stroke. 41(10): S103-s106.

[20]. Yakushiji Y, Yokota C, Yamada N, Kuroda Y, Minematsu K (2011) Clinical characteristics by topographical distribution of brain microbleeds, with a particular emphasis on diffuse microbleeds. J Stroke Cerebrovasc Dis. 20(3): 214-21.

[21]. Vernooij MW, van der Lugt A, Ikram MA, Wielopolski PA, Niessen WJ, et al., (2008) Prevalence and risk factors of cerebral microbleeds: the Rotterdam Scan Study. Neurology. 70(14): 1208-14.

[22]. Imaizumi T, Miyata K, Inamura S, Kohama I, Nyon KS, et al., (2011) The difference in location between traumatic cerebral microbleeds and microangiopathic microbleeds associated with stroke. J Neuroimaging. 21(4): 359-364.

[23]. Jeerakathil T, Wolf PA, Beiser A, Hald JK, Au R, et al., (2004) Cerebral microbleeds: prevalence and associations with cardiovascular risk factors in the Framingham Study. Stroke. 35(8): 1831-1835

[24]. Romero JR, Preis SR, Beiser A, DeCarli C, Viswanathan A, et al., (2014) Risk factors, stroke prevention treatments, and prevalence of cerebral microbleeds in the Framingham Heart Study. Stroke. 45(5): 1492-1494.

[25]. McCarron MO, Nicoll JA, Stewart J, Ironside JW, Mann DM, et al., (1999) The apolipoprotein E epsilon2 allele and the pathological features in cerebral amyloid angiopathy-related hemorrhage. J Neuropathol Exp Neurol. 58(7): 711-718

[26]. Maxwell SS, Jackson CA, Paternoster L, Cordonnier C, Thijs V, et al., (2011) Genetic associations with brain microbleeds: Systematic review and meta-analyses. Neurology. 77(2): 158-167.

[27]. Lesnik Oberstein SA, van den Boom R, van Buchem MA, van Houwelingen HC, Bakker E, et al., (2011) Cerebral microbleeds in CADASIL. Neurology. 57(6): 1066-1070.

[28]. Dichgans M, Holtmannspötter M, Herzog J, Peters N, Bergmann M, et al., (2002) Cerebral microbleeds in CADASIL: a gradient-echo magnetic resonance imaging and autopsy study. Stroke. 33(1):67-71.

[29]. Yamada M (2004) Cerebral amyloid angiopathy and gene polymorphisms. J Neurol Sci. 226(1-2): 41-44.

[30]. Biffi A, Shulman JM, Jagiella JM, Cortellini L, Ayres AM, et al., (2012) Genetic variation at CR1 increases risk of cerebral amyloid angiopathy. Neurology. 78(5): 334-341.

[31]. Lanfranconi S, Markus HS (2010) COL4A1 mutations as a monogenic cause of cerebral small vessel disease: a systematic review. Stroke. 41(8): e513-518.

[32]. Ryan NS, Bastos-Leite AJ, Rohrer JD, Werring DJ, Fox NC, et al., (2012) Cerebral microbleeds in familial Alzheimer's disease. Brain. 135(1): e201.

[33]. Reisin RC, Romero C, Marchesoni C, Nápoli G, Kisinovsky I, et al., (2011) Brain MRI findings in patients with Fabry disease. J Neurol Sci. 305(1-2): 41-44.

[34]. Schrag M, Greer DM (2014) Clinical associations of cerebral microbleeds on magnetic resonance neuroimaging. J Stroke Cerebrovasc Dis. 23(10): 2489-2497.

[35]. Zaitsu Y, Terae S, Kudo K, Tha KK, Hayakawa M, et al., (2010) Susceptibility-weighted imaging of cerebral fat embolism. J Comput Assist Tomogr. 34(1): 107-12
[36]. Klein I, Iung B, Labreuche J, Hess A, Wolff M, et al., (2009) Cerebral microbleeds are frequent in infective endocarditis: a case-control study. Stroke. 40(11): 3461-3465.

[37]. Okazaki S, Sakaguchi M, Hyun B, Nagano K, Tagaya M, et al., (2011) Cerebral microbleeds predict impending intracranial hemorrhage in infective endocarditis. Cerebrovasc Dis. 32(5): 483-488.

[38]. Ay H, Sahin G, Saatci I, Söylemezoğlu F, Saribaş O (2002) Primary angiitis of the central nervous system and silent cortical hemorrhages. AJNR Am J Neuroradiol. 23(9): 1561-1563.

[39]. Mori N, Miki Y, Kikuta K, Fushimi Y, Okada T, et al., (2008) Microbleeds in moyamoya disease: susceptibility-weighted imaging versus T2*-weighted imaging at 3 Tesla. Invest Radiol. 43(8): 574-579.

[40]. Charidimou A, Krishnan A, Werring DJ, Rolf Jäger H (2013) Cerebral microbleeds: a guide to detection and clinical relevance in different disease settings. Neuroradiology. 55(6): 655-674.

[41]. Wardlaw JM, Smith EE, Biessels GJ, Cordonnier C, Fazekas F, et al., (2013) Neuroimaging standards for research into small vessel disease and its contribution to ageing and neurodegeneration. Lancet Neurol. 12(8): 822-838.

[42]. Pantoni L (2010) Cerebral small vessel disease: from pathogenesis and clinical characteristics to therapeutic challenges. Lancet Neurol. 9(7): 689-701.

[43]. Østergaard L, Engedal TS, Moreton F, Hansen MB, Wardlaw JM, et al., (2016) Cerebral small vessel disease: Capillary pathways to stroke and cogniive decline. J Cereb Blood Flow Metab. 36(2) : 302-325.

[44]. Yamada M (2015) Cerebral amyloid angiopathy: emerging concepts. J Stroke. 17(1): 17-30.

[45]. Vinters HV (1987) Cerebral amyloid angiopathy. A critical review. Stroke 18(2): 311-324. Review.

[46]. Yamada M, Tsukagoshi H, Otomo E, Hayakawa M (1987) Cerebral amyloid angiopathy in the aged. J Neurol. 234(6): 371-376.

[47]. Samarasekera N, Smith C, Al-Shahi Salman R (2012) The association between cerebral amyloid angiopathy and intracerebral haemorrhage: systematic review and meta-analysis. J Neurol Neurosurg Psychiatry. 83(3): 275-2781.

[48]. Dierksen GA, Skehan ME, Khan MA, Jeng J, Nandigam RN, et al., (2010) Spatial relation between microbleeds and amyloid deposits in amyloid angiopathy. Ann Neurol. 68(4): 545-548.

[49]. Linn J, Halpin A, Demaerel P, Ruhland J, Giese AD, et al., (2010) Prevalence of superficial siderosis in patients with cerebral amyloid angiopathy. Neurology. 74(17): 1346-1350.

[50]. Knudsen KA, Rosand J, Karluk D, Greenberg SM (2001) Clinical diagnosis of cerebral amyloid angiopathy: validation of the Boston criteria. Neurology. 56(4): 537-539.

[51]. Kövari E, Charidimou A, Herrmann FR, Giannakopoulos P, Bouras C, et al., (2015) No neuropathological evidence for a direct topographical relation between microbleeds and cerebral amyloid angiopathy. Acta Neuropathol Commun. 3: 49

52]. Teo J, Ramadan H, Gregoire SM, Lipman G, Jäger HR, et al., (2011) Can cerebral microbleeds cause an acute stroke syndrome? Neurol Clin Pract. 1(1): 75-77.

[53]. Werring DJ, Coward LJ, Losseff NA, Jäger HR, Brown MM (2005) Cerebral microbleeds are common in ischemic stroke but rare in TIA. Neurology. 65(12): 1914-1918.

[54]. Pettersen JA, Sathiyamoorthy G, Gao FQ, Szilagyi G, Nadkarni NK, et al., (2008) Microbleed topography, leukoaraiosis, and cognition in probable Alzheimer disease from the Sunnybrook dementia study. Arch Neurol. 65(6): 790-795

[55]. Scheid R, Preul C, Gruber O, Wiggins C, von Cramon DY (2003) Diffuse axonal injury associated with chronic traumatic brain injury: evidence from T2*-weighted gradient-echo imaging at $3 \mathrm{~T}$. AJNR Am J Neuroradiol. 24(6): 1049-1056.

[56]. Lee SH, Kim BJ, Roh JK (2006) Silent microbleeds are associated with volume of primary intracerebral hemorrhage. Neurology. 66(3): 430-432.

[57]. Greenberg SM, Eng JA, Ning M, Smith EE, Rosand J (2004) Hemorrhage burden predicts recurrent intracerebral hemorrhage after lobar hemorrhage. Stroke. 35(6): 1415-1420.

[58]. Charidimou A, Kakar P, Fox Z, Werring DJ (2013) Cerebral microbleeds and recurrent stroke risk: systematic review and meta-analysis of prospective ischemic stroke and transient ischemic attack cohorts. Stroke. 44(4): 995-1001.

[59]. Akoudad S, Ikram MA, Koudstaal PJ, Hofman A, Niessen WJ, et al., (2014) Cerebral microbleeds are associated with the progression of ischemic vascular lesions. Cerebrovasc Dis. 37(5): 382-388.

[60]. Fluri F, Jax F, Amort M, Wetzel SG, Lyrer PA, et al., (2012) Significance of microbleeds in patients with transient ischaemic attack. Eur J Neurol. 19(3): 522-524.

[61]. Lim JS, Kwon HM, Lee YS (2016) Location of Cerebral Microbleeds May Predict Subsequent Stroke after Transient Ischemic Attack. J Stroke. 18(2): 
236-238.

[62]. Jeon SB, Kwon SU, Cho AH, Yun SC, Kim JS, et al., (2009) Rapid appearance of new cerebral microbleeds after acute ischemic stroke. Neurology. 73(20): 1638-1644.

[63]. Lee SH, Lee ST, Kim BJ, Park HK, Kim CK, et al., (2011) Dynamic temporal change of cerebral microbleeds: long-term follow-up MRI study. PLoS One.6(10): e25930.

[64]. Akoudad S, Portegies ML, Koudstaal PJ, Hofman A, van der Lugt A, et al., (2015) Cerebral Microbleeds Are Associated With an Increased Risk of Stroke: The Rotterdam Study. Circulation. 132(6): 509-516.

[65]. Fan YH, Zhang L, Lam WW, Mok VC, Wong KS (2013) Cerebral microbleeds as a risk factor for subsequent intracerebral hemorrhages among patients with acute ischemic stroke. Stroke. 34(10): 2459-262.

[66]. Kwa VI, Algra A, Brundel M, Bouvy W, Kappelle LJ (2013) Microbleeds as a predictor of intracerebral haemorrhage and ischaemic stroke after a TIA or minor ischaemic stroke: a cohort study. BMJ Open. 3(5): e002575.

[67]. Pasquini M, Benedictus MR, Boulouis G, Rossi C, Dequatre-Ponchelle N, et al., (2016) Incident Cerebral Microbleeds in a Cohort of Intracerebral Hemorrhage. Stroke. 47(3): 689-694.

[68]. Adams HP Jr, del Zoppo G, Alberts MJ, Bhatt DL, Brass L, et al., (2007) Guidelines for the early management of adults with ischemic stroke: a guideline from the American Heart Association/American Stroke Association Stroke Council, Clinical Cardiology Council, Cardiovascular Radiology and Intervention Council, and the Atherosclerotic Peripheral Vascular Disease and Quality of Care Outcomes in Research Interdisciplinary Working Groups: the American Academy of Neurology affirms the value of this guideline as an educational tool for neurologists. Stroke. 38(5): 1655-711.

[69]. Fiehler J, Albers GW, Boulanger JM, Derex L, Gass A, et al., (2007) Bleeding risk analysis in stroke imaging before thromboLysis (BRASIL): pooled analysis of T2*-weighted magnetic resonance imaging data from 570 patients. Stroke. 38(10): 2738-2744.

[70]. Shoamanesh A, Kwok CS, Lim PA, Benavente OR (2013) Postthrombolysis intracranial hemorrhage risk of cerebral microbleeds in acute stroke patients: a systematic review and meta-analysis. Int J Stroke. 8(5): 348-356.

[71]. Yan S, Chen Y, Zhang X, Liebeskind DS, Lou M (2014) New microbleeds after thrombolysis: contiguous thin-slice 3T MRI. Medicine. 93(20): e99.

[72]. Werring DJ (2015) Cerebral Microbleeds and Thrombolysis-Associated Intracerebral Hemorrhage: Cause for Concern, or Just a Distraction? Stroke. 46(9): 2403-2405.

[73]. Kidwell CS, Saver JL, Villablanca JP, Duckwiler G, Fredieu A, et al., (2002) Magnetic resonance imaging detection of microbleeds before thrombolysis: an emerging application. Stroke. 33(1): 95-98.

[74]. Derex L, Nighoghossian N (2008) Intracerebral haemorrhage after thrombolysis for acute ischaemic stroke: an update. J Neurol Neurosurg Psychiatry. 79(10): 1093-1099.

[75]. Kimura K, Aoki J, Shibazaki K, Saji N, Uemura J, et al., (2013) New appearance of extraischemic microbleeds on $\mathrm{T} 2{ }^{*}$-weighted magnetic resonance imaging 24 hours after tissue-type plasminogen activator administration. Stroke. 44(10): 2776-2781

[76]. Dannenberg S, Scheitz JF, Rozanski M, Erdur H, Brunecker P, et al., (2014) Number of cerebral microbleeds and risk of intracerebral hemorrhage after intravenous thrombolysis. Stroke. 45(10): 2900-2905.

[77]. Charidimou A, Kakar P, Fox Z, Werring DJ (2013) Cerebral microbleeds and the risk of intracerebral haemorrhage after thrombolysis for acute ischaemic stroke: systematic review and meta-analysis. J Neurol Neurosurg Psychiatry. 84(3): 277-280.

[78]. Turc G, Sallem A, Moulin S, Tisserand M, Machet A, et al., (2015) Microbleed Status and 3-Month Outcome After Intravenous Thrombolysis in 717 Patients With Acute Ischemic Stroke. Stroke. 46(9): 2458-2463.

[79]. Selim M, Fink JN, Kumar S, Caplan LR, Horkan C, et al., (2002) Predictors of hemorrhagic transformation after intravenous recombinant tissue plasminogen activator: prognostic value of the initial apparent diffusion coefficient and diffusion-weighted lesion volume. Stroke. 33(8): 2047-2052.

[80]. Gorelick PB, Weisman SM (2005) Risk of hemorrhagic stroke with aspirin use: an update. Stroke. 36(8): 1801-1807.

[81]. Vernooij MW, Haag MD, van der Lugt A, Hofman A, Krestin GP, et al., (2009) Use of antithrombotic drugs and the presence of cerebral microbleeds: the Rotterdam Scan Study. Arch Neurol. 66(6): 714-720.

[82]. Liu S, Li C (2015) Antiplatelet Drug Use and Cerebral Microbleeds: A Meta-analysis of Published Studies. J Stroke Cerebrovasc Dis. 24(10): 22362244.

[83]. Lovelock CE, Cordonnier C, Naka H, Al-Shahi Salman R, Sudlow CL, et al., (2010) Antithrombotic drug use, cerebral microbleeds, and intracerebral hemorrhage: a systematic review of published and unpublished studies. Stroke. 41(6): 1222-1228

[84]. Wang DN, Hou XW, Yang BW, Lin Y, Shi JP, et al., (2015) Quantity of Cerebral Microbleeds, Antiplatelet Therapy, and Intracerebral Hemorrhage
Outcomes: A Systematic Review and Meta-analysis. J Stroke Cerebrovasc Dis. 24(12): 2728-2737.

[85]. Gregoire SM, Jäger HR, Yousry TA, Kallis C, Brown MM, et al., (2010) Brain microbleeds as a potential risk factor for antiplatelet-related intracerebral haemorrhage: hospital-based, case-control study. J Neurol Neurosurg Psychiatry. 81(6): 679-684.

[86]. Leys D, Cordonnier C (2010) Brain microbleeds as a potential risk factor for antiplateletrelated intracerebral haemorrhage. J Neurol Neurosurg Psychiatry. 81(6): 589-590.

[87]. Wong KS, Chan YL, Liu JY, Gao S, Lam WW (2003) Asymptomatic microbleeds as a risk factor for aspirin-associated intracerebral hemorrhages. Neurology. 60(3): 511-513.

[88]. Imaizumi T, Inamura S, Kohama I, Yoshifuji K, Nomura T, et al., (2014) Nascent deep microbleeds and stroke recurrences. J Stroke Cerebrovasc Dis. 23(3): 520-528.

[89]. Darweesh SK, Leening MJ, Akoudad S, Loth DW, Hofman A, et al., (2013) Clopidogrel use is associated with an increased prevalence of cerebral microbleeds in a stroke-free population: the Rotterdam study. J Am Heart Assoc. 2(5): e000359.

[90]. Naka H, Nomura E, Kitamura J, Imamura E, Wakabayashi S, et al., (2013) Antiplatelet therapy as a risk factor for microbleeds in intracerebral hemorrhage patients: analysis using specific antiplatelet agents. J Stroke Cerebrovasc Dis. 22(6): 834-840.

[91]. Kim CK, Kwon HT, Kwon HM (2012) No significant association of aspirin use with cerebral microbleeds in the asymptomatic elderly. J Neurol Sci. 319(1-2): 56-58.

[92]. Orken DN, Uysal E, Timer E, Kuloglu-Pazarcı N, Mumcu S, et al., (2013) New cerebral microbleeds in ischemic stroke patients on warfarin treatment: two-year followup. Clin Neurol Neurosurg. 115(9): 1682-1685.

[93]. Thijs V, Lemmens R, Schoofs C, Görner A, Van Damme P, et al., (2010) Microbleeds and the risk of recurrent stroke. Stroke. 41(9): 2005-2009.

[94]. Akoudad S, Darweesh SK, Leening MJ, Koudstaal PJ, Hofman A, et al., (2014) Use of coumarin anticoagulants and cerebral microbleeds in the general population. Stroke. 45(11): 3436-3439.

[95]. Charidimou A, Shakeshaft C, Werring DJ (2012) Cerebral microbleeds on magnetic resonance imaging and anticoagulant-associated intracerebral hemorrhage risk. Front Neurol. 3: 133.

[96]. Lee SH, Ryu WS, Roh JK (2012) Cerebral microbleeds are a risk factor for warfarin-related intracerebral hemorrhage. Neurology. 72(2): 171-176.

[97]. Hart RG, Diener HC, Yang S, Connolly SJ, Wallentin L, et al., (2012) Intracranial hemorrhage in atrial fibrillation patients during anticoagulation with warfarin or dabigatran: the RE-LY trial. Stroke. 43(6): 1511-1517.

[98]. Ruff CT, Giugliano RP, Braunwald E, Hoffman EB, Deenadayalu N, et al., (2014) Comparison of the efficacy and safety of new oral anticoagulants with warfarin in patients with atrial fibrillation: a meta-analysis of randomised trials. Lancet. 383(9921): 955-962.

[99]. Connolly SJ, Ezekowitz MD, Yusuf S, Eikelboom J, Oldgren J, et al., (2009) Dabigatran versus warfarin in patients with atrial fibrillation. $\mathrm{N}$ Engl J Med. 361(12): 1139-1151.

[100]. Granger CB, Alexander JH, McMurray JJ, Lopes RD, Hylek EM, et al. (2011) Apixaban versus warfarin in patients with atrial fibrillation. N Eng J Med. 365(11): 981-992.

[101]. Patel MR, Mahaffey KW, Garg J, Pan G, Singer DE, et al., (2011) Rivaroxaban versus warfarin in nonvalvular atrial fibrillation. $N$ Engl J Med. 365(10): 883-891

102]. Saito T, Kawamura Y, Sato N, Kano K, Takahashi K, et al., (2015) Non-vitamin $\mathrm{k}$ antagonist oral anticoagulants do not increase cerebral microbleeds. J Stroke Cerebrovasc Dis. 24(6): 1373-1377.

[103]. Steinberg BA, Piccini JP (2014) Anticoagulation in atrial fibrillation. BMJ. 348: g2116.

[104].Eckman MH, Rosand J, Knudsen KA, Singer DE, Greenberg SM (2003) Can patients be anticoagulated after intracerebral hemorrhage? A decision analysis. Stroke. 34(7): 1710-1716.

[105]. Haley KE, Greenberg SM, Gurol ME (2013) Cerebral microbleeds and macrobleeds: should they influence our recommendations for antithrombotic therapies? Curr Cardiol Rep. 15(12): 425

[106]. Wilson D, Jäger HR, Werring DJ (2015) Anticoagulation for Atrial Fibrillation in Patients with Cerebral Microbleeds. Curr Atheroscler Rep. 17(8): 47.

[107]. Wu R, Feng C, Zhao Y, Jin AP, Fang M, et al., (2014) A meta-analysis of association between cerebral microbleeds and cognitive impairment. Med Sci Monit. 20: 2189-2198.

[108]. Pantoni L, Poggesi A, Inzitari D (2009) Cognitive decline and dementia related to cerebrovascular diseases: some evidence and concepts. Cerebrovasc Dis. 27(1): 191-196.

[109]. Yamashiro K, Tanaka R, Okuma Y, Shimura H, Ueno Y, et al., (2014) Cerebral microbleeds are associated with worse cognitive function in the 
nondemented elderly with small vessel disease. Cerebrovasc Dis Extra. 4(3): 212-220.

[110]. Yakushiji Y, Nishiyama M, Yakushiji S, Hirotsu T, Uchino A, et al., (2008) Brain microbleeds and global cognitive function in adults without neurological disorder. Stroke. 39(12): 3323-3328.

[111]. Van Norden AG, van den Berg HA, de Laat KF, Gons RA, van Dijk EJ, et al., (2011) Frontal and temporal microbleeds are related to cognitive function: the Radboud University Nijmegen Diffusion Tensor and Magnetic Resonance Cohort (RUN DMC) Study. Stroke. 42(12): 3382-3386.

[112]. Akoudad S, Wolters FJ, Viswanathan A, de Bruijn RF, van der Lugt A, et al., (2016) Association of Cerebral Microbleeds With Cognitive Decline and Dementia. JAMA Neurol. 73(8): 934-943.

[113]. Cordonnier C, van der Flier WM, Sluimer JD, Leys D, Barkhof F, et al., (2006) Prevalence and severity of microbleeds in a memory clinic setting. Neurology. 66(9): 1356-1360.

[114]. Kim TW, Lee SJ, Koo J, Choi HS, Park JW, et al., (2014) Cerebral microbleeds and functional outcomes after ischemic stroke. Can J Neurol Sci. 41(5): 577-582.

[115]. Brown WR, Thore CR (2011) Review: cerebral microvascular pathology in ageing and neurodegeneration. Neuropathol Appl Neurobiol. 37(1): 56-74.
[116].Strbian D, Sairanen T, Meretoja A, Pitkäniemi J, Putaala J, et al., (2011) Patient outcomes from symptomatic intracerebral hemorrhage after stroke thrombolysis. Neurology. 77(4): 341-348.

[117]. Cai J, Fu J, Yan S, Hu H, Lin C (2015) Clinical Outcome in Acute Ischemic Stroke Patients With Microbleeds After Thrombolytic Therapy: A MetaAnalysis. Medicine. 94(52): e2379.

[118].Altmann-Schneider I, Trompet S, de Craen AJ, van Es AC, Jukema JW, et al., (2011) Cerebral microbleeds are predictive of mortality in the elderly. Stroke. 42(3): 638-644.

[119]. Henneman WJ, Sluimer JD, Cordonnier C, Baak MM, Scheltens P, et al., (2009) MRI biomarkers of vascular damage and atrophy predicting mortality in a memory clinic population. Stroke. 40(2): 492-498.

[120]. Charidimou A, Wilson D, Shakeshaft C, Ambler G, White M, et al., (2015) The Clinical Relevance of Microbleeds in Stroke study (CROMIS-2): rationale, design, and methods. Int J Stroke. 100: 155-161.

[121]. Takizawa S, Tanaka F, Nishiyama K, Hasegawa Y, Nagata E, et al., (2015) Protocol for Cerebral Microbleeds during the Non-Vitamin K Antagonist Oral Anticoagulants or Warfarin Therapy in Stroke Patients with Nonvalvular Atrial Fibrillation (CMB-NOW) Study: Multisite Pilot Trial. J Stroke Cerebrovasc Dis. 24(9): 2143-2148. 